\title{
Establishment of an Orthotopic Xenograft Mice Model of Retinoblastoma Suitable for Preclinical Testing
}

\author{
Nathalie Cassoux ${ }^{a}$ b Aurélie Thuleau $^{b}$ Franck Assayag $^{b}$ Isabelle Aerts $^{c}$ \\ Didier Decaudin ${ }^{b}, d$ \\ a Département d'oncologie chirurgicale, 'baboratory of Preclinical Investigation, \\ Department of Translational Research, 'Département d'oncologie pédiatrique de \\ I'adolescent et de l'adulte jeune (DOPAJA), and d Department of Medical Oncology, \\ Institut Curie, Paris, France
}

\section{Key Words}

Retinoblastoma $\cdot$ Xenograft rodent models $\cdot$ Histological analysis $\cdot$ Patient-derived xenografts

\begin{abstract}
Retinoblastoma is a rare cancer that occurs during childhood. The goal of current and future therapeutic strategies is to conserve the eye and visual function without using external beam radiotherapy, which is known to increase the risk of secondary cancers in genetically predisposed patients. Multimodality therapy (usually intravenous but also intra-arterial and intravitreal chemotherapy, transpupillary thermotherapy, cryotherapy, or brachytherapy) has recently improved the eye salvage rate in retinoblastoma and has led to a decreased need for external beam radiotherapy. However, the treatment of advanced intraocular retinoblastoma remains a real challenge, especially in cases of vitreous and subretinal seeding. There is a need for alternative and less toxic therapies as well as for better ways to administer the drugs. Animal models are an integral part of preclinical research in the field of oncology. This paper describes the different xenograft rodent models published in the literature so far. We will also describe a new orthotopic xenografted retinoblastoma model in immunodeficient mice, which is suitable for preclinical assays. The xenograft model was established from tumor tissue obtained directly from surgical samples and closely mimics human retinoblastoma.
\end{abstract}




\section{Ocular Oncology \\ and Pathology}

\begin{tabular}{l|l}
\hline Ocul Oncol Pathol 2015;1:200-206 \\
\hline DOI: $10.1159 / 000370156$ & $\begin{array}{l}\text { @ 2015 S. Karger AG, Basel } \\
\text { www.karger.com/oop }\end{array}$ \\
\hline
\end{tabular}

Cassoux et al.: Establishment of an Orthotopic Xenograft Mice Model of Retinoblastoma Suitable for Preclinical Testing

\section{Introduction}

Retinoblastoma is a rare childhood cancer of the retina with 250-300 cases per year in the United States, 1/15,000-20,000 live births in France and 6,000-8,000 per year worldwide [1]. When left untreated, the disease is lethal, but survival reaches more than $95 \%$ with the appropriate treatment. Both heritable and sporadic forms of retinoblastoma result from inactivation of both alleles of the retinoblastoma susceptibility gene RB1. Children who inherit one defective copy of $R B 1$ are likely to develop bilateral multifocal retinoblastoma as a result of inactivation of the second $R B 1$ allele in the retina. Children who present with two spontaneous $R B 1$ mutations in the same retinal cell during their development will have a unilateral unifocal retinoblastoma tumor. The majority of unilateral sporadic retinoblastoma cases are diagnosed at an advanced stage of the disease and are enucleated to minimize the risk of metastasis. Children with less advanced unilateral retinoblastoma or bilateral retinoblastoma undergo conservative therapy (at least in one eye in bilateral cases) to avoid enucleation or blindness. Chemotherapy combined with transpupillary thermotherapy for posterior tumors, plus cryotherapy and brachytherapy for anterior tumors, has improved the external radiotherapy-free eye salvage rate and preserved useful vision [2]. However, advanced bilateral retinoblastoma remains difficult to treat. Tumor cell invasion of the vitreous and subretinal fluid remains one of the challenging issues due to the poor diffusion of drugs into the eye. Following the pioneering work of Stallard and Reese, external beam radiotherapy was used for more than 20 years to treat bilateral retinoblastoma or vitreous seeding in the remaining eye. However, after this treatment, retinoblastoma patients have a significant risk of developing a second cancer, especially sarcoma, in the irradiated field [2,3]. During recent years, considerable efforts have been made to avoid external beam irradiation as much as possible through the use of systemic chemotherapy. New techniques such as intra-arterial chemotherapy or intravitreal injections of chemotherapeutic agents have been developed with the same goal [4-6].

There is a need for alternative and less toxic therapies as well as for new ways of local administration of the drugs, for instance through the development of delivery devices or iontophoresis. In vitro studies play an important role in the preclinical screening of new drugs but have their own limitations as they are being conducted outside the complex microenvironment and organ stroma that exist inside the human body. Hence, animal models are an integral part of preclinical research in the field of oncology. Nonhuman tumor models (syngeneic models) have helped to understand the course of tumorigenesis and to evaluate therapeutic protocols in several human cancers. Another approach that has been successful for rare tumors such as medulloblastomas or retinoblastomas consists of patient-derived xenografts that are currently used in the preclinical therapeutic screening of drugs in a majority of cancers (breast, lung, or colorectal cancer, cutaneous melanoma, etc.) [7]. The main advantage of these models for the preclinical screening of drugs is the immediate access to the tumor and the easiness to measure the tumor's response to drugs using calipers, whereas the main disadvantages of these models are the absence of interaction with both the original stroma and immune system since the rodents used are immunosuppressed animals. This is especially problematic when tumors that grow in an organ with 'immune privilege', with a closed blood-aqueous barrier as seen in the eyeball, are studied [8]. In recent years, considerable efforts have been made to develop more clinically relevant models by using orthotopic transplantations of human tumor material in rodents. The implantation of tumor cells into the organ of origin (orthotopically) allows organotypical interactions between tumor cells and the surrounding stroma. It has been shown that tumor cell interaction with the environment affects the growth, differentiation and drug sensitivity of tumor cells. Moreover, tumor cells can spread to metastatic sites in other organs, with specificities comparable to those in humans [9]. 
Cassoux et al.: Establishment of an Orthotopic Xenograft Mice Model of Retinoblastoma Suitable for Preclinical Testing

In this review, we present the orthotopic intraocular models that we have recently developed. Transgenic mouse models will not be discussed in this paper.

\section{Orthotopic Retinoblastoma Models}

Orthotopic intraocular retinoblastoma models have been developed by injecting human retinoblastoma cells directly into the anterior chamber or the vitreous cavity of immunodeficient mice or by using cells from subcutaneous patient-derived xenografts [10]. The human cells that have been used widely in such models are Y79 and WERI-Rb retinoblastoma cells, which were first generated in the 1970s and came from the American Type Culture Collection (Manassa, Va., USA) [11]. Although these tumor cells may not reflect a patient's tumor heterogeneity, many teams used these cell lines to evaluate different drugs or ways for administering the drugs. One of the earliest studies was done by McFall et al. [11], who injected a suspension of Y79 and WERI-Rb cells into the anterior chamber of immunosuppressed rabbits, which resulted in tumor growth. Chévez-Barrios et al. [12] described in detail two orthotopic models using Y79 and WERI-Rb cells, which were injected into the vitreal cavity using a Hamilton syringe. The authors showed that 2 weeks after the injection, tumor seeds started to grow in the vitreous, and after 5 weeks, the whole eye was invaded. In this paper, only Y79 orthotopic xenografts showed metastatic spread to the brain [12]. The main disadvantage of this model is that the tumor developed in the vitreous cavity instead of starting as a retinal tumor. Other teams used the same route to inject tumor cells in adult immunodeficient mice or in newborn rats $[13,14]$.

Another inconvenience is the lack of a measurable mass on clinical examination. Several researchers used bioluminescence imaging to monitor tumor growth [15]. Kang and Grossniklaus [16] developed a rabbit model using WERI-Rb cells injected in the subretinal space. They reported that the tumor was detectable by fundoscopy with progressive vascularization of the tumor.

At the Institut Curie in Paris, Aerts et al. [17] developed a panel of retinoblastoma patientderived xenografts placed subcutaneously in adult nude mice (Swiss nu/nu) using tumor tissue obtained directly from surgical samples. These models were evaluated histologically as well as with genomic analysis and showed a good correlation between the patient's tumor characteristics and the xenograft. The validity of the model for drug testing was determined by using an intraperitoneal therapeutic standard regimen with a combination of carboplatin and etoposide. Tumor evolution was measured with calipers. All tumors showed a significant response to the applied combination of chemotherapy compared to the untreated control group. This model was then used to evaluate the efficiency of photodynamic therapy, which showed promising results for tumor growth. However, these results are not comparable with the clinical situation since the treated tumors were not inside the eye [17].

We developed orthotopic models of retinoblastoma using the panel of xenografted tumors previously described by Aerts et al. [17] that were maintained by serial passages from one mouse to another or were frozen. Briefly, mice bearing subcutaneous xenografted tumors were sacrificed by cervical dislocation. After tumor excision, a suspension of fresh cells was prepared in culture medium. Adult immunodeficient SCID mice were used for the experiment. Animals were handled in accordance with the Association for Research in Vision and Ophthalmology (ARVO) statement for the use of animals in ophthalmology and research in vision. Under intraperitoneal general anesthesia with sodium pentobarbital, the pupil was dilated with one drop of tropicamide, after which a drop of topical anesthetic proparacaine hydrochloride $(0.5 \%)$ was applied. Injections were delivered using a surgical microscope; $2 \mu \mathrm{l}$ of cell suspension $(10,000$ cells $/ \mu \mathrm{l})$ was injected into the subretinal space of the right eye using a Hamilton syringe with a 33-gauge needle (fig. 1). 
Ocular Oncology

and Pathology
Ocul Oncol Pathol 2015;1:200-206

DOI: $10.1159 / 000370156$

Cassoux et al.: Establishment of an Orthotopic Xenograft Mice Model of Retinoblastoma Suitable for Preclinical Testing

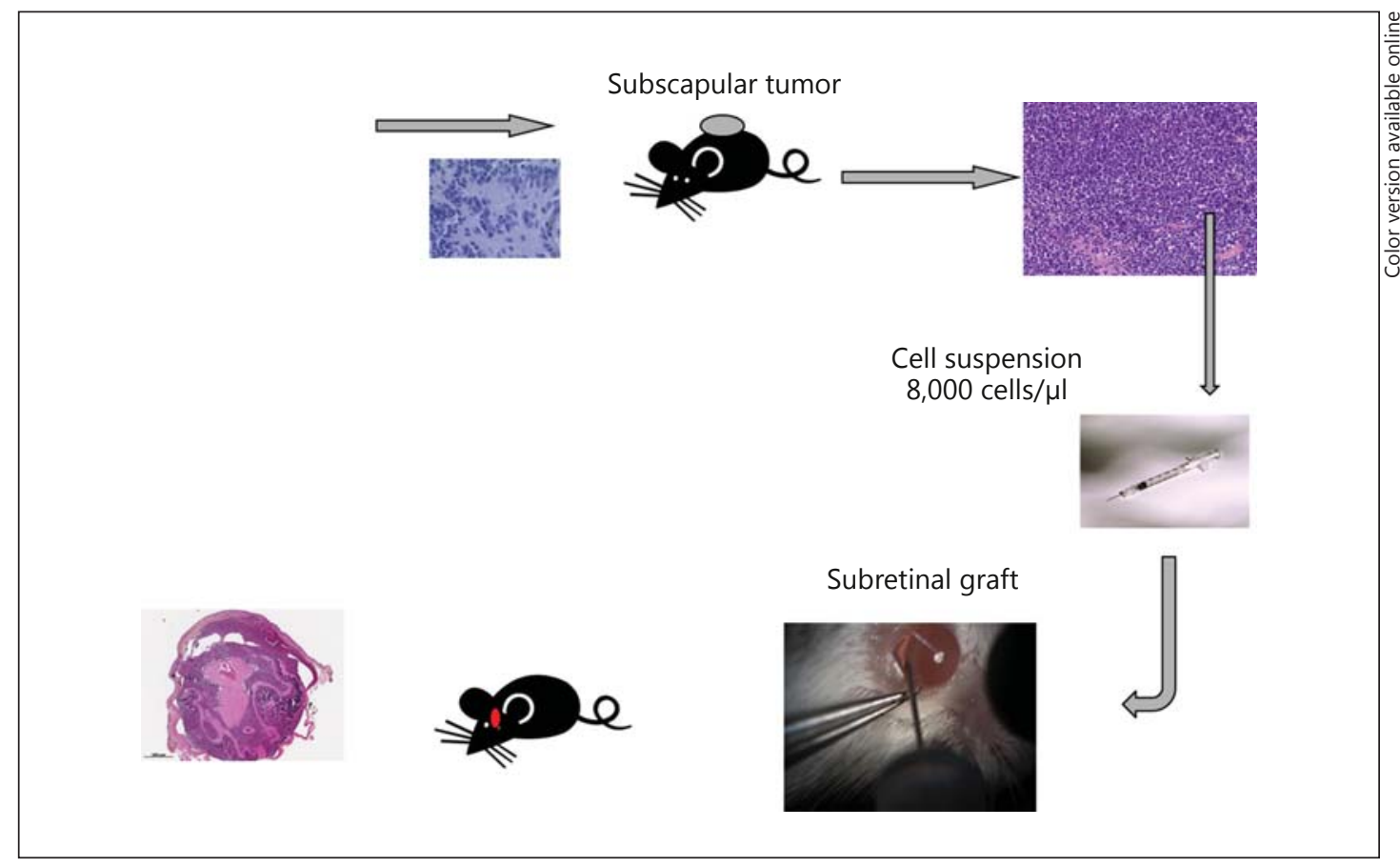

Fig. 1. Orthotopic xenograft procedure.

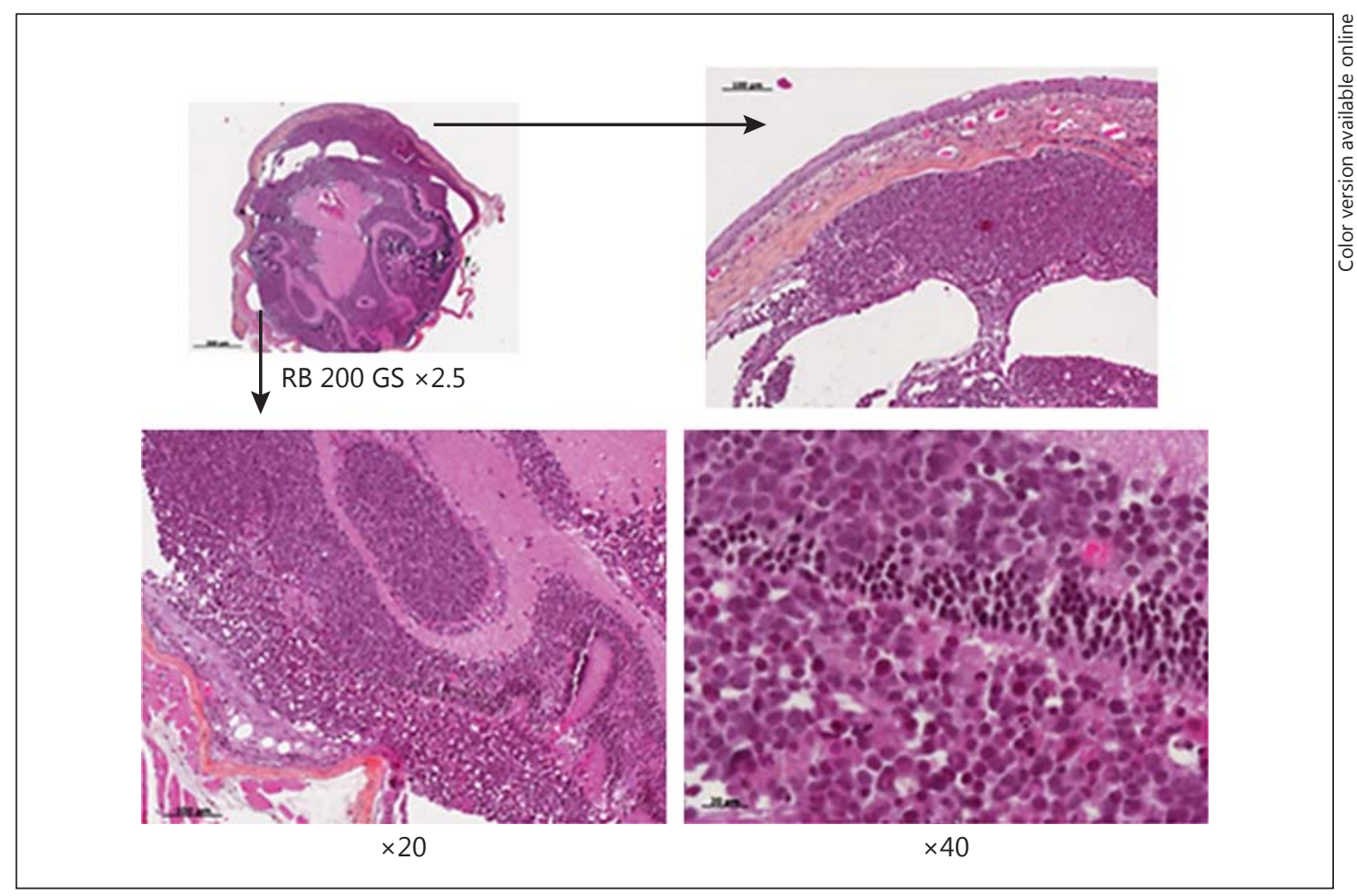

Fig. 2. Histological analysis of a mouse eye showing a massive infiltration of the retina as well as the vitreous and anterior chambers by retinoblastoma tumor cells. 
Ocular Oncology

and Pathology
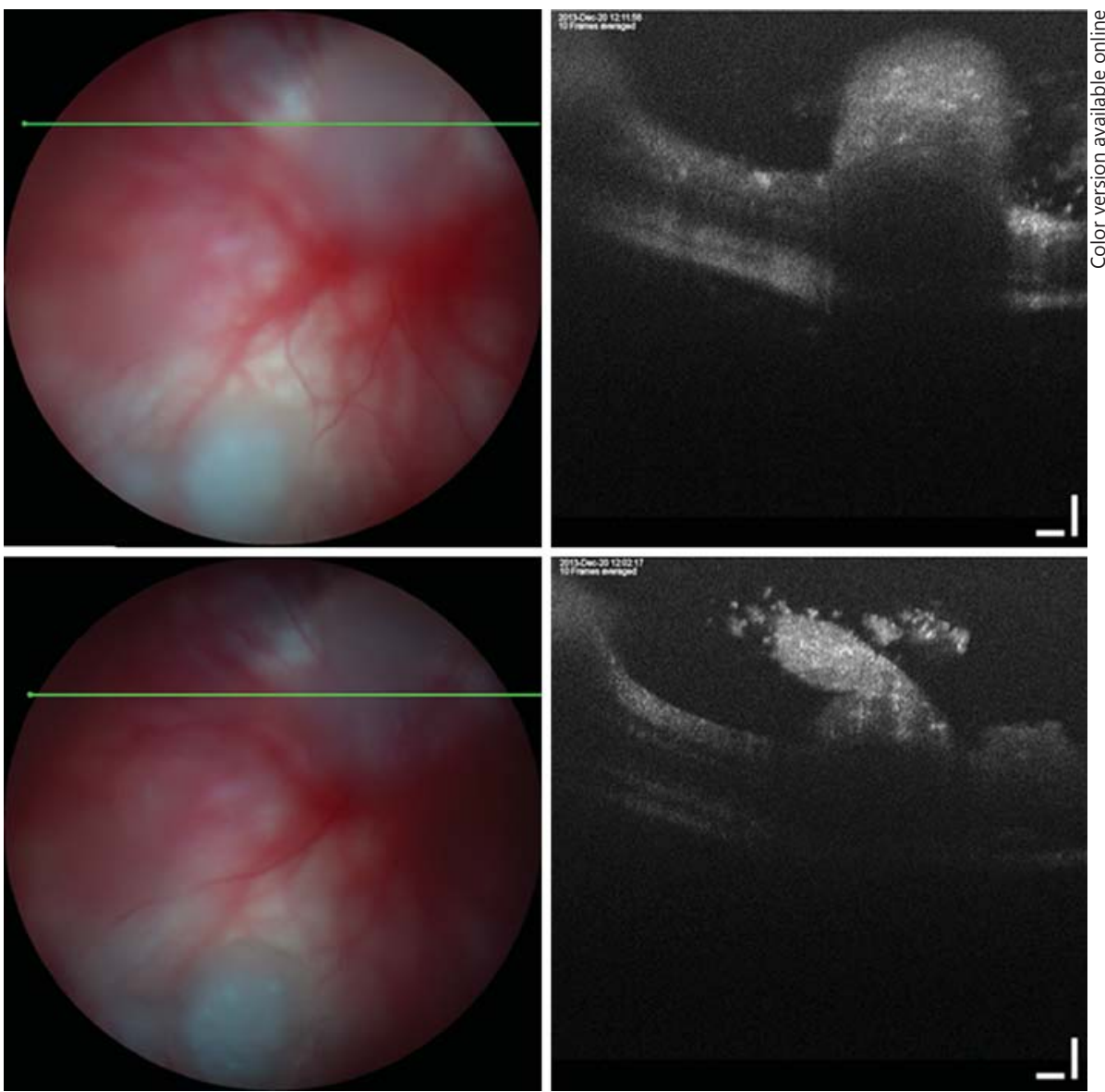

Fig. 3. Fundus photography and OCT of mouse eyes after orthotopic xenograft of retinoblastoma showing two retinal tumors; one retinal tumor starts to invade the vitreous (images using Micron IV, Phoenix Research Labs).

After subretinal injection, ophthalmic examination of the mice was performed weekly. Clinical findings regarding the presence of a retinal tumor or a vitreal invasion were recorded. Retinal images and optical coherence tomography (OCT) were used to monitor tumor growth using the Micron IV (Phoenix Research Labs, USA). When tumor cells invaded the vitreous cavity, mice were sacrificed by cervical dislocation for histological analyses. The eyes were immediately fixed in $10 \%$ formalin and then embedded in paraffin using conventional systems. The blocks were sectioned to obtain 4- to 5- $\mu$ m-thick sections and stained with conventional hematoxylin-eosin (fig. 2). During monitoring, we observed uni- or bifocal retinal tumors with progressive invasion of the vitreous cavity. The development of retinal tumors was demonstrated by OCT (fig. 3).

Tumors developed in all injected eyes 4-6 weeks after orthotopic transplantation. In the first two xenografts, the proliferation started with a white retinal mass; 3 weeks later, the vitreous was invaded by tumor seeds that extended into the anterior chamber. Pathological examination of the injected eyes confirmed the presence of a massive infiltration of the retina as well as the vitreous and anterior chambers by retinoblastoma cells.

The model was assessed by a therapeutic experiment evaluating the efficacy of intravitreal injections of melphalan, carboplatin and bevacizumab and saline as control. One of our xenografts, Rb-200, was selected for the study. Four groups of 6 SCID mice received a xeno- 
Ocular Oncology

and Pathology

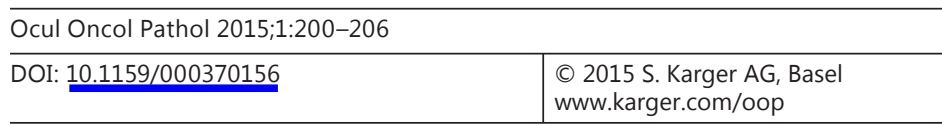

Cassoux et al.: Establishment of an Orthotopic Xenograft Mice Model of Retinoblastoma Suitable for Preclinical Testing

graft in the subretinal space according to our protocol. Ophthalmic examination was performed weekly, and intravitreal injections were started when tumors began to invade the vitreous body. Intravitreal injections were performed once a week for 4 weeks, after which time the mice were sacrificed for histological analysis. Histological analysis disclosed massive tumor infiltration in $100 \%$ of the eyes treated with bevacizumab or saline solution. Melphalan was able to clear $33 \%$ of the eyes and carboplatin cleared $83 \%$ of the eyes; intravitreal injection of carboplatin was more efficient than that of melphalan. This work was presented at the ARVO meeting in 2011 [18].

The limitation of our model is the same as for all xenografted models, orthotopic or not, and is related to the lack of a functioning immune system and the artificial graft of human tumor cells in an ectopic site. Even when the grafts were placed orthotopically in the eye, the cells were implanted in the subretinal space, which does not exactly follow the physiopathology of the development of retinoblastoma in humans.

\section{Conclusion}

The ideal retinoblastoma animal model that exhibits all features of the human form does not exist to date, and xenografted rodent models remain a good tool for the therapeutic screening of new drugs, for studying new ways of drug administration (iontophoresis), new devices (sustained-release implants, charged nanoparticles) or alternative therapies (dynamic phototherapy) $[17,19,20]$. The placement of human retinoblastoma xenografts under the retina provides a model that can be used effectively to screen potential drugs for the treatment of retinoblastoma.

\section{Disclosure Statement}

The authors declare that there are no conflicts of interest.

\section{References}

1 Aerts I, Lumbroso-Le Rouic L, Gauthier-Villars M, Brisse H, Doz F, Desjardins L: Retinoblastoma. Orphanet J Rare Dis 2006;1:31.

2 Lumbroso-Le Rouic L, Aerts I, Levy-Gabriel C, et al: Conservative treatments of intraocular retinoblastoma. Ophthalmology 2008;115:1405-1410.

-3 Marees T, van Leeuwen FE, Schaapveld M, et al: Risk of third malignancies and death after a second malignancy in retinoblastoma survivors. Eur J Cancer 2010;46:2052-2058.

$\$ 4$ Munier FL, Gaillard MC, Balmer A, et al: Intravitreal chemotherapy for vitreous disease in retinoblastoma revisited: from prohibition to conditional indications. Br J Ophthalmol 2012;96:1078-1083.

5 Abramson DH: Retinoblastoma: saving life with vision. Annu Rev Med 2014;65:171-184.

-6 Shields CL, Kaliki S, Rojanaporn D, Al-Dahmash S, Bianciotto CG, Shields JA: Intravenous and intra-arterial chemotherapy for retinoblastoma: what have we learned? Curr Opin Ophthalmol 2012;23:202-209.

7 Romer JT, Curran T: Medulloblastoma and retinoblastoma: oncology recapitulates ontogeny. Cell Cycle 2004; 3:917-919.

-8 McKenna KC, Kapp JA: Ocular immune privilege and CTL tolerance. Immunol Res 2004;29:103-112.

$\rightarrow 9$ Bibby MC: Orthotopic models of cancer for preclinical drug evaluation: advantages and disadvantages. Eur J Cancer 2004;40:852-857.

10 Benedict WF, Dawson JA, Banerjee A, Murphree AL: The nude mouse model for human retinoblastoma: a system for evaluation of retinoblastoma therapy. Med Pediatr Oncol 1980;8:391-395.

-11 McFall RC, Sery TW, Makadon M: Characterization of a new continuous cell line derived from a human retinoblastoma. Cancer Res 1977;37:1003-1010.

$\checkmark 12$ Chévez-Barrios P, Hurwitz MY, Louie K, et al: Metastatic and nonmetastatic models of retinoblastoma. Am J Pathol 2000;157:1405-1412. 
Cassoux et al.: Establishment of an Orthotopic Xenograft Mice Model of Retinoblastoma Suitable for Preclinical Testing

13 Dyer MA, Rodriguez-Galindo C, Wilson MW: Use of preclinical models to improve treatment of retinoblastoma. PLoS Med 2005;2:e332.

14 Laurie NA, Gray JK, Zhang J, et al: Topotecan combination chemotherapy in two new rodent models of retinoblastoma. Clin Cancer Res 2005;11:7569-7578.

15 Ji X, Cheng L, Wei F, et al: Noninvasive visualization of retinoblastoma growth and metastasis via bioluminescence imaging. Invest Ophthalmol Vis Sci 2009;50:5544-5551.

16 Kang SJ, Grossniklaus HE: Rabbit model of retinoblastoma. J Biomed Biotechnol 2011;394730.

17 Aerts I, Leuraud P, Blais J, et al: In vivo efficacy of photodynamic therapy in three new xenograft models of human retinoblastoma. Photodiagnosis Photodyn Ther 2010;7:275-283.

18 Cassoux N, Assayag F, Nemati F, Fontaine JJ, Chateau-Joubert S, Aerts I, Doz F, Desjardins L, Decaudin D: Development of new models of orthotopic primary human retinoblastoma xenografts. ARVO Meeting 2011, abstract 1591.

19 Nemeth KM, Federico S, Carcaboso AM, et al: Subconjunctival carboplatin and systemic topotecan treatment in preclinical models of retinoblastoma. Cancer 2011;117:421-434.

20 Tsui JY, Dalgard C, Van Quill KR, et al: Subconjunctival topotecan in fibrin sealant in the treatment of transgenic murine retinoblastoma. Invest Ophthalmol Vis Sci 2008;49:490-496. 